experiment shows that the planes of polarization of two photons are always perpendicular, it means $\pi_{S}=-1$, and the only possibility left is (4).

The author would like to thank all those who discussed with him one or the other of the subject matters dealt with in this work, thus indirectly helping him to prepare this survey article. In particular, he is grateful to Professor E. P. Wigner for many enlightening discussions. Apologies are extended to the authors whose contributions to the field of problems treated in this paper have not been quoted at the appropriate places. $^{35}$

${ }^{35}$ Among many others: E. P. Wigner and L. Eisenbud, Phys. Rev. 72, 29 (1947); W. Pauli, lecture notes, Summer School of Theoretical Physics, Les Houches, 1952; F. Coester, Phys. Rev. 84, 1259 (1951); F. Coester, Phys. Rev. 89, 619 (1953); L. Michel, Nuovo Cimento, 10, 320 (1953); G. Lüders, Kongel. Danske Vidensk. Selsk. Mat. fys. Medd. 28, 1 (1954); Umezawa, Kamefuchi, and Tanaka, Soryushi-Ron-Kenkyu (in Japanese) 6, 543, 1954; the same authors, Prog. Theoret. Phys. (to be printed); Y. Katayama, Soryushi-Ron-Kenkyu (in Japanese) 6, 543 (1954); E. R. Caianiello, Physica 18, 1020 (1952); and other papers of the same author; S. Oneda, Prog. Theoret. Phys. 9, 327 (1953).

REVIEWS OF MODERN PHYSICS

VOI, UME 27, NUMBER 1

J A N U A R Y, 1955

\title{
Erratum: The Energy Levels and the Structure of Light Nuclei
}

\author{
D. R. InGLIS \\ Argonne National Laboratory, Lemont, Illinois \\ [Revs. Modern Phys. 25, 390 (1953)]
}

Figure 9 is in error in having the low ${ }^{2} P$ plotted four units too low and is to be replaced by the accompanying figure. This removes what might have been considered a reason for doubting recent experimental suggestions ${ }^{1}$ that there may be excited states of $\mathrm{Be}^{9}$ near 1.8 and $3.1 \mathrm{Mev}$, indicated by dotted lines in the inserts of the figure, in addition to the well-established $2.43-\mathrm{Mev}$ state recently observed $^{2}$ to have $J \geqslant \frac{3}{2}$ as now appears consistent with this rough exploratory treatment of the theory. The error was discovered and very kindly pointed out by Edith Halbert and Sudhir Pandya of the University of Rochester, who are calculating the intermediate-coupling transition of the lowest states of several $(J, T)$.

1 Kunz, Moak, and Good, Phys. Rev. 95, 640 (1954) ; F. Ajzenberg and T. Lauritsen, Revs. Modern Phys. 27, 91 (1955) which quotes K.jW. Allen on $\mathrm{B}^{10}(l, \alpha) \mathrm{Be}^{9}$.

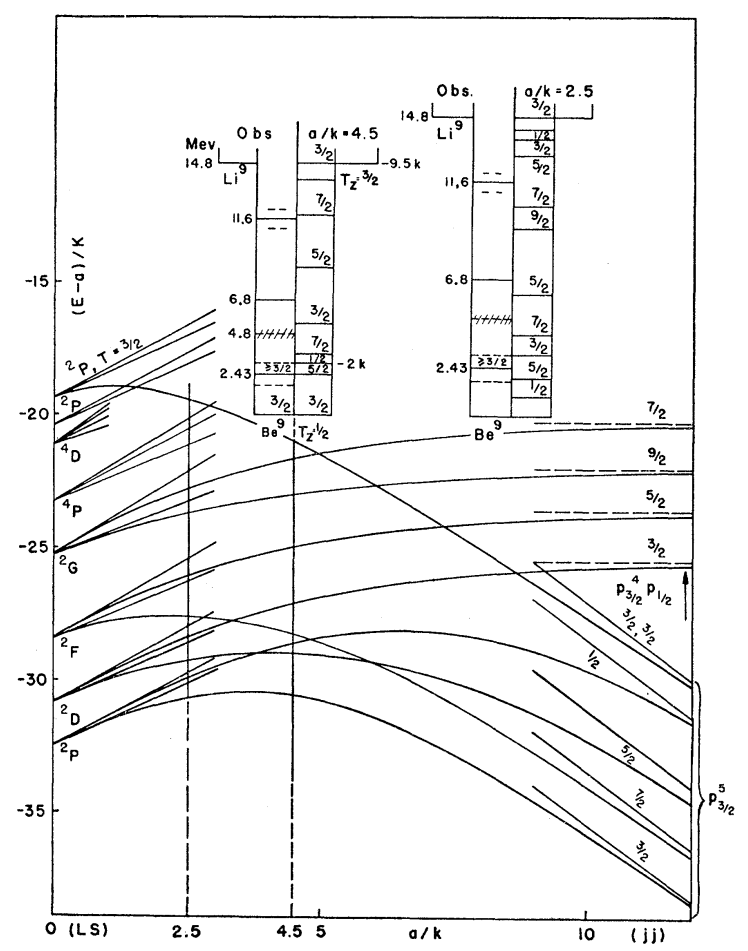

Fig. 9. Intermediate-coupling transition for the configuration $p^{5}$ applying to $\mathrm{Py}^{9}$. The isobaric spin is $T=\frac{1}{2}$ except where indicated as $T=\frac{3}{3}$. 